\title{
Open approaches for cruciate ligament reconstruction in knee dislocations: A technical note and case series
}

\author{
Michael Held ${ }^{1, *}$, Martiz Laubscher ${ }^{1}$, Richard von Bormann ${ }^{2}$, Dustin L. Richter ${ }^{3}$, Daniel C. Wascher ${ }^{3}$, \\ and Robert C. Schenck ${ }^{3}$ \\ ${ }^{1}$ Department of Orthopaedic Surgery, Groote Schuur Hospital, Orthopaedic Research Unit, University of Cape Town, 7925 Cape Town, \\ South Africa \\ 2 Knee Unit, Groote Schuur Hospital and Christiaan Barnard Memorial Hospital, University of Cape Town, 7700 Cape Town, South \\ Africa \\ 3 Department of Orthopaedics \& Rehabilitation, The University of New Mexico Health Sciences Center, Albuquerque, 87131-0001 NM, \\ USA
}

Received 15 September 2020, Accepted 28 February 2021, Published online 22 March 2021

\begin{abstract}
Introduction: Arthroscopic surgery is the gold standard for cruciate ligament reconstruction in multiligament knee injuries. However, hospitals in limited-resource settings often lack arthroscopic-trained surgeons or equipment. Open approaches for treating knee dislocations can overcome many of these limitations. Methodology: This study aims to describe techniques for open approaches in a supine patient to address the cruciate ligaments in multiligament knee injuries and to review associated complications and clinical outcomes in a retrospective case series. Results: Ten patients with multi-ligament knee injuries who had undergone open cruciate ligament reconstruction between July 2016 and November 2018 were retrospectively identified. Open approaches were performed owing to the extravasation of arthroscopy fluid into the posterior compartment (3) or a large traumatic arthrotomy (7). Complications and patient-reported outcomes were analysed. Eight of the 10 patients were followed up at 10 months postoperatively (range, 5-23 months). None had iatrogenic neurovascular damage. Median outcomes scores were: visual analogue scale, 45 (range, 0-100); Knee Injury and Osteoarthritis Outcome Score-Physical Function Short Form, 81.4 (range, 75-100); Lysholm, 85 (range, 67-92). Discussion: Open approaches were safe and useful in treating cruciate ligaments and should be considered in arthroscopy fluid extraversion and large traumatic arthrotomies.
\end{abstract}

Key words: Knee dislocation, Multi-ligament knee injury, Multiple ligamentous injuries, Open approach, Limited resource settings (LRS).

\section{Introduction}

For multi-ligament knee injuries (MLKIs), most authors promote an arthroscopic reconstruction of cruciate ligaments and open surgical treatment of lateral and medial structures to achieve good outcomes [1-4]. Arthroscopy can also help to assess and treat associated meniscal and cartilaginous injuries, decrease the risk of arthrofibrosis, and result in less injury to the articular cartilage.

But the risk of arthroscopy, especially in acute MLKIs, is fluid extravasation and the concomitant risk of compartment syndrome or vascular compromise. Also, in knee dislocations with a large Morel-type lesion in which traumatic dissection can be used to access ligaments (Figure 1), arthroscopy is poten-

*Corresponding author:michael.held@uct.ac.za tially unnecessary. In some circumstances, such as an irreducible knee dislocation, an open approach is the only safe initial approach [5-7]. Thus, even surgeons well versed in arthroscopic techniques need to be familiar with alternatives such as open approaches for cruciate ligament reconstruction. Open approaches for treating knee dislocations can overcome many of these limitations and allow surgeons to stabilize the knee without the need for specialized arthroscopic equipment or skills.

There is a paucity of studies on open cruciate reconstruction from state hospitals in a limited resource setting (LRS). Yet, these centres often lack arthroscopic-trained surgeons and equipment for which open techniques play a vital role to provide trauma care for a large patient population [8]. Also, a comprehensive description of open access to cruciate ligaments through various approaches in a single publication is not available. 
The purpose of this study was to present open approaches used to address both cruciate ligaments in patients with MLKIs. Our aims were as follows: (1) describe surgical approaches that can be useful to treat patients with acute injuries and in hospitals in LRS, and (2) report short-term clinical outcomes of ten patients with knee dislocations treated with open cruciate ligament reconstruction by a single surgeon (MH) in an LRS.

\section{Methods}

The primary aim of this work is to provide a technical note for open approaches in a supine patient to address the cruciate ligaments in multi-ligament knee injuries. Furthermore, a case series of patients treated with these techniques were reviewed retrospectively to describe associated complications and clinical outcomes.

\section{Surgical technique}

Patients are positioned supine to allow full knee flexion and extension. A radiolucent table enables fluoroscopy if necessary. A foot bolsters placed under the heel keeps the knee flexed $70^{\circ}$, allowing easier access to the tibial footprint of the anterior cruciate ligament (ACL). With the toes on the bolster, knee flexion can be increased to allow easier access to the femoral insertion of the cruciate ligaments. A high side bolster prevents the leg from rotating at the hip. Tourniquets, when used, are placed as proximal as possible to allow insertion of guidewires without compromise.

\section{Incision}

The primary incision is curvilinear anteromedial beginning halfway between the medial border of the patella and the medial epicondyle, extending distally along the anteromedial surface of the tibia to below the pes anserine attachment. This incision allows access to the intercondylar notch, the proximal and distal attachments of the medial collateral ligament, and the tibial insertion of the PCL (i.e., Lobenhoffer approach, Video 1). It also allows harvesting of the patella tendon and hamstring tendons and could be extended proximally for a quadriceps tendon harvest. For lateral side injuries, the lateral incision begins over the lateral epicondyle and extended distally posterior and inferior to the fibular head. This incision gives access to the peroneal nerve, the posterolateral structures, and the PCL tibial attachment. It also facilitates outside-in drilling and fixation of the ACL femoral attachment.

The decision of whether to approach the posterior part of the knee laterally or medially is dictated by the collateral ligament involvement. This is usually confirmed via magnetic resonance imaging or stress radiographs. The approach to the PCL through the medial Lobenhoffer interval is facilitated by traumatic dissection of posteromedial structures and capsules. Access through the Lobenhoffer interval is performed by the takedown of the semimembranosus muscle as needed, and then using the plane between the medial collateral ligament (MCL) and medial head of the gastrocnemius with an elevation of the popliteus muscle. Similarly, posterolateral corner (PLC)

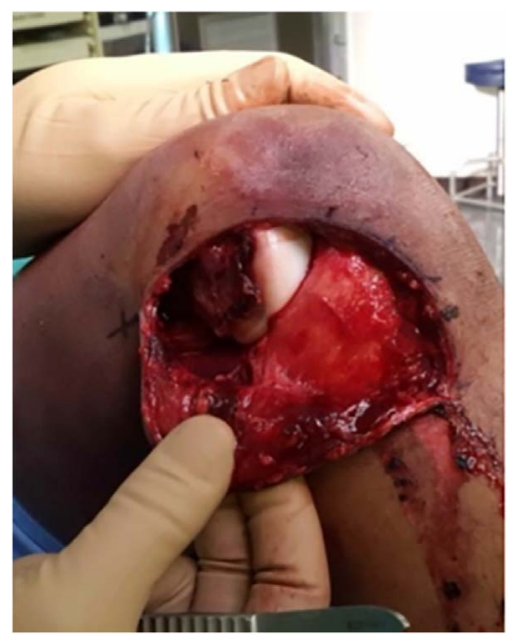

Figure 1. Large medial capsular tear with exposed medial condyle after skin incision in an irreducible knee dislocation. This allows access to the tibial insertion of the posterior cruciate ligament through the traumatic arthrotomy without extensive surgical dissection.

injuries facilitated the approach to the PCL through a lateral incision [9].

\section{Notch access}

Access to the notch is achieved through a medial parapatellar dissection (Figure 2, Video 2). This can be extended subvastus, midvastus, or proximally into the quadriceps tendon to enable patella sublaxation, greatly increasing exposure [10]. Although the fat pad may be retained [11, 12], it is frequently debulked to increase visualization. The cruciate ligament stumps are excised to allow better visualization of the insertion sites for guide pin placement. The knee is placed in slight extension to enable visualization of the tibial insertion of the ACL, as well as the anterior horns and roots of the menisci. It also facilitated the placement of the ACL tibial and PCL femoral tunnels by removing tension off the extensor mechanism. If cruciate ligament guides are available, the use of Z-retractors and fat pad excision can provide acceptable visualization of the notch even in more limited incisions. A headlamp can provide clearer visualization.

Guide pins for the femoral attachments for the ACL and PCL are placed in an "inside-out" fashion. When performing a single bundle PCL reconstruction, the guide pin is started in the centre of the anterolateral bundle. The ACL pin is started at the centre of the ACL femoral footprint. The ACL tibial guide pin is placed in an "outside-in" fashion.

\section{Medial approach}

The posterior aspect of the knee can be approached through the Lobenhoffer interval [9, 13]. The superficial dissection should expose the hamstrings and MCL. To increase access, the knee was flexed to $90^{\circ}$ with the ipsilateral hip externally rotated. Visualization is improved by working from the 


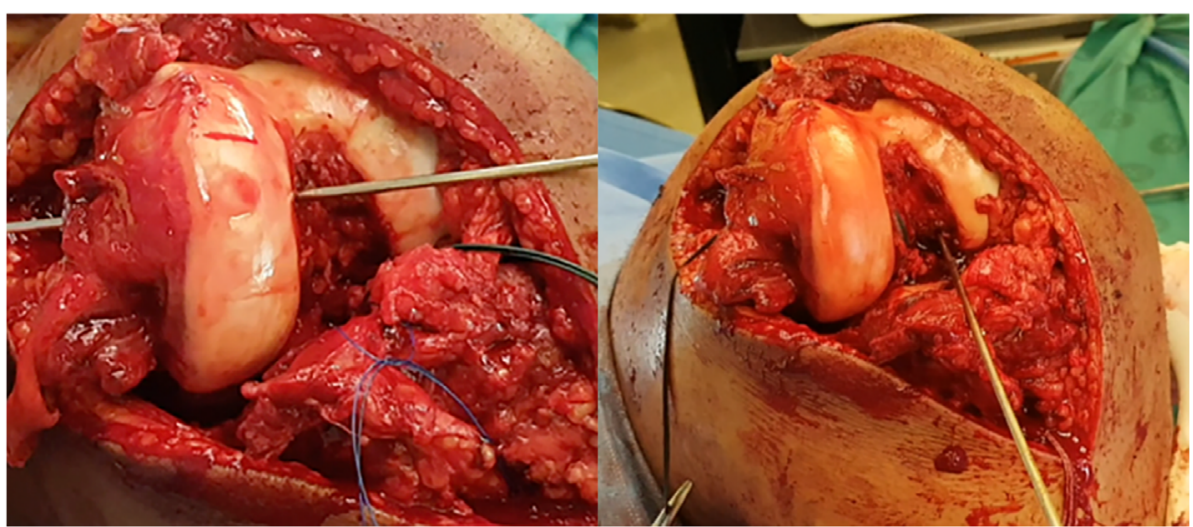

Figure 2. Intraoperative access to the notch of left through a medial parapatellar dissection with the patella retracted. This gives appropriate access to important structures in the notch. Left: guidewire drilled through the femoral footprint of the anterolateral bundle of the posterior cruciate ligament. Right: guidewire drilled through the femoral footprint of the anterior cruciate ligament.
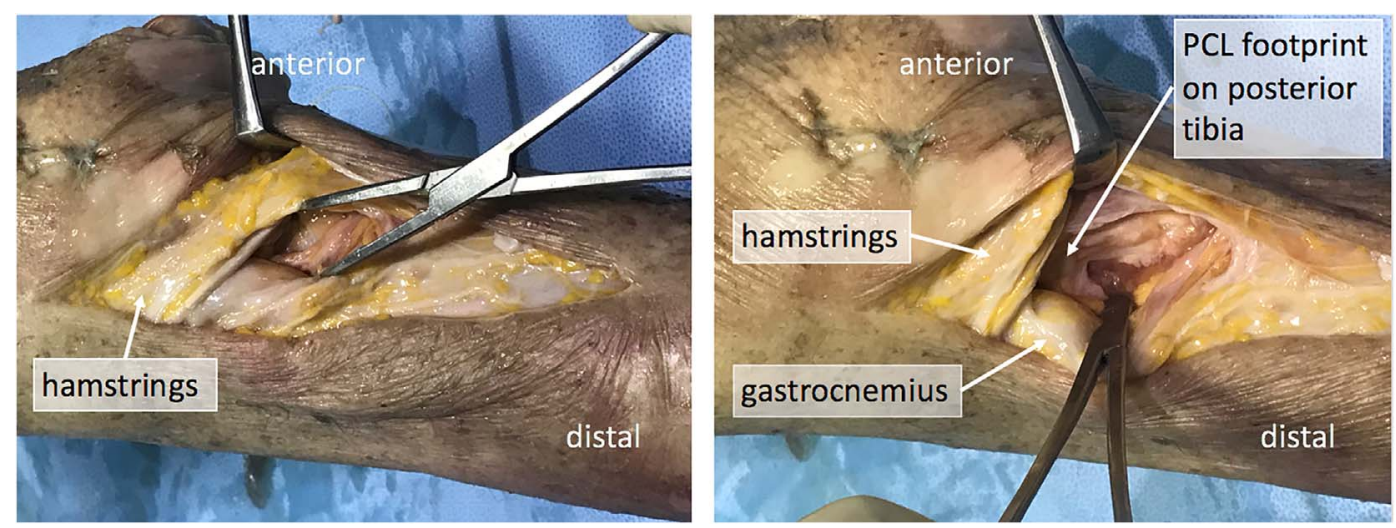

Figure 3. Medial Lobenhoffer approach in a left cadaveric knee. Left: the haemostat shows access to the posterior part of the knee, inferior to the attachments of the hamstrings. Right: the gastrocnemius muscle is reflected posteriorly to give access to the posterior capsule and posterior cruciate ligament. Note the haemostat is spreading fibres of the popliteus muscle. Leaving the pes intact and working above and below the pes in knee flexion allows for safe access to the back of the tibia.

opposite side of the table. A sponge can be used to clean residual fat off these structures to identify the proximal and distal border of the pes anserine.

For acute injuries to the posteromedial corner, the traumatic dissection often enables palpation of the champagne glass dropoff and PCL stump around the posteromedial part of the knee. To gain further access, a Hohman retractor is placed anterior to the medial head of the gastrocnemius muscle.

External rotation of the tibia and blunt dissection of the fibres of the popliteus muscle gives access to the posterior capsule and PCL tibial insertion (Figure 3, Video 3). Working with a blunt instrument, such as a Cobb (Skylar Surgical Instruments, West Chester, Pennsylvania), the residual capsule was stripped until full access and visualization of the PCL insertion is possible. It is critical to keep the retractors anterior to the gastrocnemius and popliteal muscles, hugging the posterior surface of the tibia to protect the neurovascular structures during tibial tunnel drilling or through creation. Knee flexion to $60-90^{\circ}$ during dissection of the back of the tibia relaxes the neurovascular structures. Adhesions and scarring in chronic injuries can make the anatomic differentiation of structures challenging.
Use of a Cobb elevator, electrocautery, identifications of anatomic landmarks of the medial joint line and the inner edge of the posteromedial femoral condyle, and exposure through the Lobenhoffer interval allow improved visualization and safe access to the posterior knee in these multiple trauma patients. This helps avoid prone positioning and subsequent physiologic risk to already compromised patients.

\section{Lateral approach}

Using a direct lateral incision, access to the posterior aspect of the tibia and insertion of the PCL is achieved (Figure 4, Video 4). This has been described for the reconstruction of the PLC [14-17]. The incision passes just anterior to the lateral condyle and distally just posterior to the fibular head. With a separate anteromedial incision to access the notch, a sufficient skin bridge of at least $8 \mathrm{~cm}$ is maintained to avoid skin necrosis. Three fascial "windows" are created to gain access to the posterolateral structures [17].

An incision is first made posterior to the biceps tendon (window 1) to identify the peroneal nerve about $1 \mathrm{~cm}$ distal 

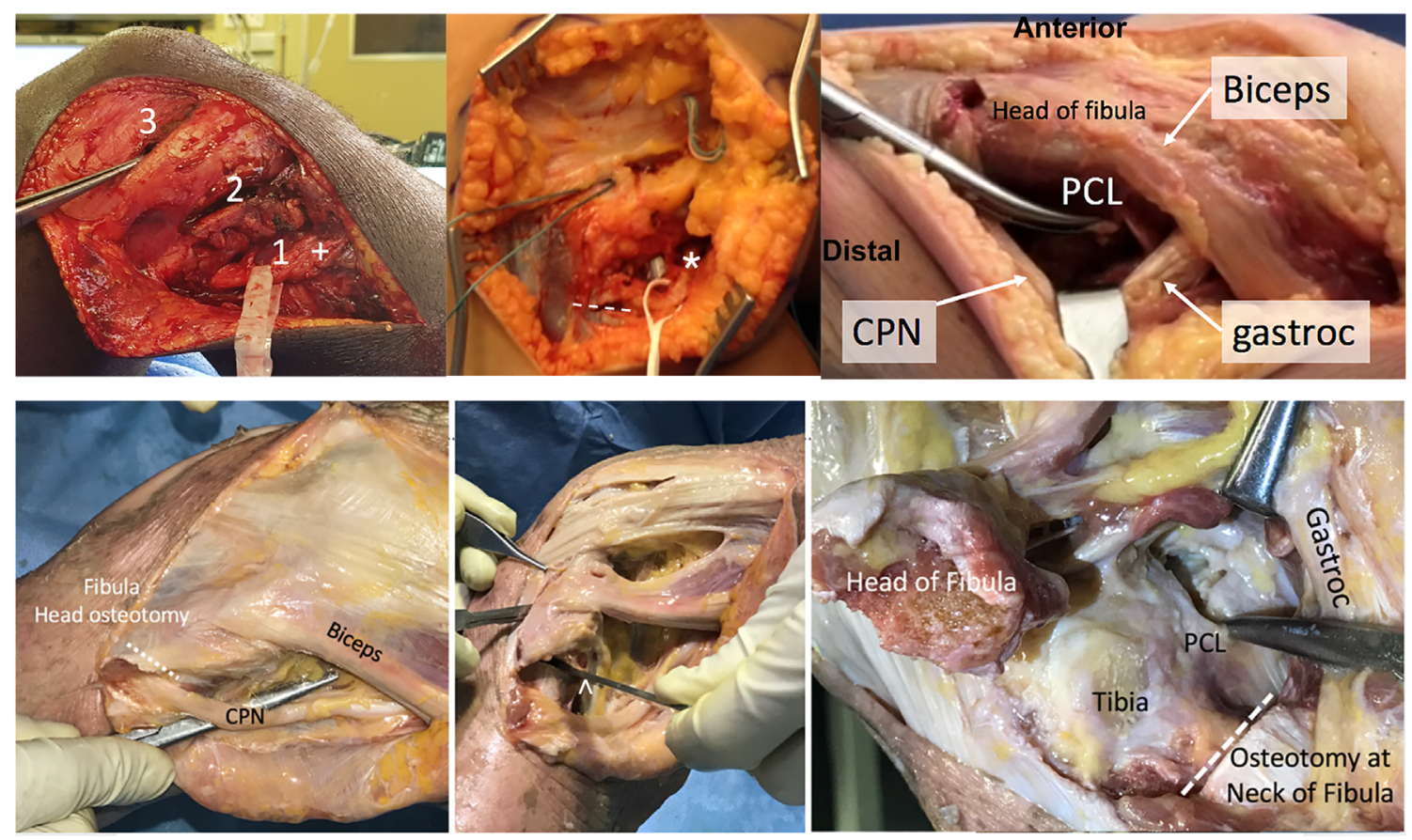

Figure 4. Lateral approach to careful dissection and retraction of common peroneal nerve (CPN). Top left: release of the nerve through window " 2 ", in which the nerve has (+) a drop-down appearance with appropriate release below window "1" and the forceps are placed into window "3". Top middle: release of peroneal nerve (*) in a chronic condition with extensive scarring and release to peroneus longus fascia (white line). Top right: lateral Lobenhoffer approach without fibula head osteotomy in a cadaver, in which protecting the CPN can be achieved through access distal to the biceps tendon, retracting the gastrocnemius ("gastroc") tendon posterior. The tibial insertion of the posterior cruciate ligament ("PCL") can be reached posterior to the head of the fibula. Bottom left: lateral Lobenhoffer approach to the posterior part of the tibia in a cadaver. Initially, the CPN is identified and protected. Bottom middle and right: An osteotomy at the neck of the fibula $\left({ }^{\wedge}\right)$ increases access to the PCL when the gastrocnemius and popliteus muscles are retracted posteriorly.

and posterior to the tip of the fibular head or just distal around the fibular neck. The nerve is released distally into the anterior compartment with the careful division of the overlying muscle fascia to achieve a tension-free "drop-down sign" of the nerve. Careful dissection and protection of the nerve throughout the procedure are crucial.

Window 2 is created next by developing the interval between the iliotibial band and the biceps femoris tendon. This window gave access to the posterolateral capsule, the lateral meniscus, the fibular collateral ligament (FCL) attachment, and the attachment of the popliteofibular ligament.

Finally, window 3 is created by splitting the iliotibial band fibres overlying the lateral epicondyle. This allowed visualization of the femoral attachments of the FCL and popliteus tendon.

The posterior tibia is accessed through the lateral approach [9]. Window 1 or 2 is used to gain access posterior to the fibular head. The lateral head of the gastrocnemius is identified and retracted posteriorly with a Hohman retractor by sliding under the popliteus tendon beneath the lateral head of the gastrocnemius muscle. Blunt dissection is used to avoid venous bleeders. A fibular neck osteotomy can be used at the metaphysealdiaphyseal junction to increase access [9]. However, with traumatic tears of the lateral collateral ligament and popliteus tendon, the exposed tibia can often be palpated around the fibular head and osteotomy is usually not necessary [18].
The tibial insertion of the PCL can be exposed by internal rotation of the tibia and placing a Hohmann retractor past the midline of the tibia, just proximal to the champagne glass drop-off. Careful posterior retraction and knee flexion to $90^{\circ}$ will protect neurovascular structures and enable appropriate access and visualization.

\section{Closure}

Medially and laterally, the interval of the posterior approach is mainly intermuscular, and no deep sutures are necessary for closure. On the lateral side, window 1 should be allowed to heal without repair to avoid any compression or injury to the peroneal nerve. The interval between the biceps femoris and iliotibial band can be reapproximated with an absorbable suture. The window 3 splits of the iliotibial band should be closed with strong absorbable braided sutures.

Medially, no suture of deep structures is necessary [9]. The popliteus muscle is split in line with the fibres during the medial approach and does not require approximation. Postoperatively, it is critical to monitor limb perfusion and peripheral nerve function. Lastly, tourniquet time must be monitored, and continuous ischemia longer than $120 \mathrm{~min}$ should be avoided. It is often difficult to perform bicruciate and lateral collateral ligament reconstructions in less than $2 \mathrm{~h}$. Surgeons should either allow reperfusion for at least $15 \mathrm{~min}$ followed by tourniquet 
Table 1. Categorization of knee dislocations according to Schenck classification, and description of associated injuries.

\begin{tabular}{|c|c|c|c|c|c|}
\hline Patient no. & Sex & Age (years) & $\mathrm{KDC}$ & Associated injuries & Associated IA pathology \\
\hline 1 & Male & 38 & KDIIIM & - & Medial meniscus bucket handle tear \\
\hline 2 & Female & 37 & KDIV & - & $\begin{array}{l}\text { Medial and lateral meniscus posterior } \\
\text { root avulsion, lateral meniscus tear } \\
\text { bucket handle }\end{array}$ \\
\hline 3 & Male & 59 & KDI & - & - \\
\hline 4 & Male & 31 & KDIV & - & - \\
\hline 5 & Male & 64 & KDIIIL & Femur fracture, closed head injury, CPN & Arcuate fracture \\
\hline 6 & Male & 58 & KDV & $\begin{array}{l}\text { Open book pelvic injury, ipsilateral } \\
\text { foot fracture, contralateral MLKI, CPN }\end{array}$ & Patella tendon rupture \\
\hline 7 & Male & 17 & KDII & - & - \\
\hline 8 & Male & 32 & KDIIIM & - & - \\
\hline 9 & Female & 33 & KDIIIL & $\mathrm{CPN}$ & - \\
\hline 10 & Male & 25 & KDIIIM & $\begin{array}{l}\text { Contralateral MLKI with CPN, } \\
\text { traumatic aortic dissection }\end{array}$ & $\begin{array}{l}\text { Medial meniscus anterior } \\
\text { root avulsion }\end{array}$ \\
\hline
\end{tabular}

Abbreviations: KDC, knee dislocation classification; IA, intraarticular; CPN, common peroneal nerve; MLKI, multi-ligament knee injury; CPNP, common peroneal nerve palsy; -, not applicable.

re-inflation or perform the remainder of the procedure (or the entire operation) without tourniquet control.

\section{Case series}

For this case series, consecutive patients who had undergone surgery for MLKI between July 2016 and November 2018 were retrospectively identified. All patients who had an open cruciate reconstruction were included. Patients below the age of 18 years were excluded. Demographic data, injury mechanism, and classification, associated injuries, time delay to surgery, as well as the indication for open surgery were collected.

The primary outcome measure was the presence of any major complications such as neurovascular injury, stiffness, delayed wound healing, or infection. Secondary patientreported outcome measures (PROMs) included the following: the visual analogue scale (VAS), Knee Injury and Osteoarthritis Outcome Score-Physical Function Short Form (KOOS-PS), and Lysholm scale.

All procedures performed in studies involving human participants were in accordance with the ethical standards of the institutional and/or national research committee (HREC REF 050/2018) and with the 1964 Helsinki declaration and its later amendments or comparable ethical standards.

\section{Results}

Ten patients (two female) with a mean age of 35 years (range: 18-64, IQR: 21.5) were identified. All injuries, except one (ultra-low energy fall), were high-energy injuries caused by road traffic collisions. Injuries were further categorized by the Schenck classification system. Eight patients had a KDIII or higher injury and most had associated fractures or soft tissue and neurovascular injuries (Table 1). All procedures but one were performed within 3 months of injury.

Three patients were converted to open surgical treatment owing to fluid extravasation during diagnostic arthroscopy.
All of these patients underwent a posteromedial approach to the posterior cruciate ligament (PCL) tibial insertion. In seven patients, a large traumatic arthrotomy enabled sufficient access to the posterior tibia through the medially based injuries (five patients) and laterally based injuries (two patients). Two patients had bilateral MLKIs, of which only one side was treated through an open approach.

Eight of the 10 patients were able to be contacted for a median follow-up of 24 months (range, 17-33 months; IQR, 20.5). No iatrogenic damage to neurovascular structures occurred.

Three patients developed arthrofibrosis with decreased flexion of less than $80^{\circ}$. Two of these patients showed heterotopic ossification on radiographs (Figure 5). One of these patients improved after manipulation under anaesthesia to $100^{\circ}$, whereas the other two patients opted not to have a further intervention. In one patient with a KDV and ipsilateral patella tendon rupture, impaired wound healing necessitated reoperation after 6 weeks, which resolved after removal of the protective cerclage wire.

PROMs were assessed at the time of final follow-up. The KOOS-PS median score was 81.4 (range, 75-100; IQR, 12.2) and the median Lysholm score was 85 (range, 67-92; IQR, 13.3). The median VAS pain rating was 45 (range, 0-50; IQR 15).

\section{Discussion}

Arthroscopic single-stage surgery is the gold-standard for cruciate reconstruction in multi-ligament knee injuries. But, open cruciate surgery avoids fluid extravasation and can overcome challenges faced by the hospital with limited arthroscopy-trained surgeons or equipment in an LRS (Table 2). The findings of this study show that open cruciate surgery in 10 patients with severe MLKIs resulted in acceptable PROMs, with an acceptable incidence of complications. Notably, arthrofibrosis and heterotopic ossification (HO) occurred in three patients.

This study has several limitations. Although the short-term follow-up allows assessment of complications, long-term 


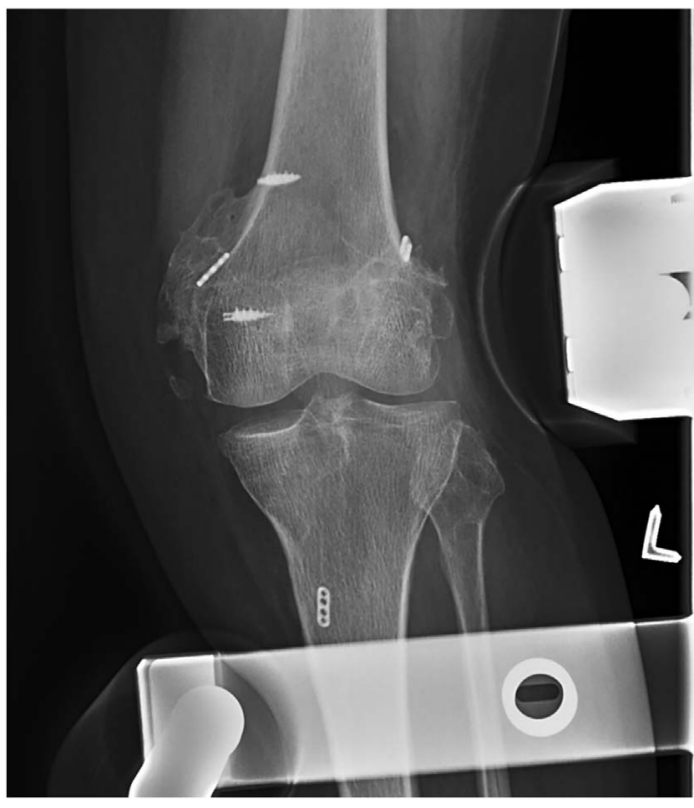

Figure 5. Radiographic valgus stress view of a patient with a KDIIIM, showing heterotopic ossification around the medial epicondyle at 9 months postoperatively.

follow-up is necessary to understand the risk of increased posttraumatic osteoarthritis [19]. Because of the low-resource setting and difficulty contacting patients, we were unable to perform any instrumented ligamentous testing. But the follow-up was sufficient to assess perioperative complications and describe a safe technique to perform this procedure. Other limitations include the retrospective design, small number of patients, and lack of a standardized treatment protocol, all of which are common challenges in knee dislocation research.

Our preferred approach is an extended anteromedial incision for bicruciate injuries involving the medial side (KDIIIM). A lateral incision is included if the PLC structures are injured (KDIII-L) or when all ligaments are affected (KDIV).
Alternatively, an anterior midline incision can be used to address all cruciate and collateral ligaments, thus avoiding multiple scars and potential wound healing problems; however, it requires extensive undermining of the skin to reach the collateral ligaments and tibial attachment of the PCL. Furthermore, accessing and protecting the peroneal nerve during PLC reconstruction can be challenging.

Other authors have described an open approach to the PCL tibial insertion with similar outcomes but this was usually done in a prone patient [7, 21-23]. The described approach allows maximum visualization of the PCL tibial footprint while protecting the neurovascular structures. Our technique can be performed in supine patients, enabling improved access to other injuries in patients with multiple traumas. Although most cruciate ligament reconstructions are performed arthroscopically [24], acceptable long-term follow-up can be achieved after open reconstruction [25]. Furthermore, a detailed summary of results of studies using open PCL [26] and ACL reconstructions [27] has been made in a meta-analysis and no difference in PROMs or complications was found when compared to arthroscopic surgery. Also, reported stiffness and HO after arthroscopic or closed treatment of knee dislocations are similar [19, 20].

Another important point to consider is that preoperative imaging is important to confirm lateral or medial laxity. This dictates the approach to the posterior part of the knee. MRI has high diagnostic accuracy in acute knee dislocations [28], but when MRI is not available, similar accuracy can be achieved with comparative clinician-assisted varus and valgus stress radiographs in $20^{\circ}$ of flexion [29-31]. Here the tibiofemoral distance at the medial or lateral joint line is measured in millimetres and compared to the contralateral side. A similar accuracy has also been found with PCL stress views. These can be done with posteriorly directed force by the clinician, or in form of kneeling radiographs [32]. Here the line of the posterior tibial cortex is referenced to the most posterior point of the Blumensaat line and this distance is compared to the contralateral side. A further advantage of stress radiographs is their dynamic component of assessment and the possibility to grade the laxity

Table 2. Key points for open cruciate procedures.

\begin{tabular}{ll}
\hline No. & \multicolumn{1}{c}{ Description } \\
\hline 1 & Use traumatic dissection of posterolateral and posteromedial corners to gain access to the PCL insertion of the tibia \\
2 & Minimize tourniquet use to prevent prolonged ischemia \\
3 & For the retraction of posterior structures, stay subperiosteal and dissect past the midline prior to placing the Hohmann retractor \\
4 & Even a small shift or subluxation of the patella out of the notch increases access and visualization of the cruciate ligaments greatly \\
5 & Decreasing flexion of the knee allows better access to the notch \\
6 & Sit during the dissection of the posterior approach to the tibia or stand on the opposite side of the table \\
7 & Rotation of the tibia is often increased in collateral ligament injuries and facilitates access to the posterior tibia
\end{tabular}

Table 3. Evaluation of posterior, varus, and valgus knee instability using stress radiographs [3].

\begin{tabular}{lcccccc}
\hline Kneeling stress & Injury & Grade & Varus stress & Injury & Valgus stress & Injury \\
\hline$\leq 7 \mathrm{~mm}$ & Normal or partial tear & I & $\leq 2.6 \mathrm{~mm}$ & Normal or partial tear & $\leq 3.1 \mathrm{~mm}$ & Normal or partial tear \\
$8-11 \mathrm{~mm}$ & Complete PCL tear & II & $2.7-3.9 \mathrm{~mm}$ & Isolated LCL tear & $3.2-9.7 \mathrm{~mm}$ & Complete sMCL tear \\
$\geq 12 \mathrm{~mm}$ & Combined ligament injury & III & $\geq 4 \mathrm{~mm}$ & Complete PLC injury & $\geq 9.8 \mathrm{~mm}$ & Complete tear of all medial structures \\
\hline
\end{tabular}

LCL, lateral collateral ligament; PCL, posterior collateral ligament; PLC, posterolateral corner; PTT, posterior tibial translation; sMCL, superficial medial collateral ligament. 
based on the distance measured (Table 3). Although preoperative assessment prior to surgery is essential for adequate planning, stress radiographs done under anaesthesia and before the incision can therefore provide crucial information even if MRI is available.

Overall, our findings support the concept that open approaches to knee dislocations can be a useful tool for cruciate ligament reconstruction in special circumstances. The most important future potential for it is the management of MLKIs in LRS.

\section{Supplementary materials}

Supplementary material is available at https://www.sicot-j. org/10.1051/sicotj/2021016/olm

Video 1. Posteromedial approach cadaver JSICOT.

Video 2. Notch access JSICOT.

Video 3. Posterolateral approach cadaver JSICOT.

Video 4. Posteromedial approach surgery JSICOT.

Acknowledgements. The authors thank Sahar Freedman for editorial assistance.

\section{Conflict of interest}

DW receives funding from Smith \& Nephew and Axogen in the form of educational grants unrelated to the submission. MH receives funding from Smith \& Nephew in the form of educational grants unrelated to the submission. ML, RvB, $\mathrm{DR}$, and RS certified that they have no financial conflict of interest (e.g., consultancies, stock ownership, equity interest, patent/licensing arrangements, etc) in connection with this article.

\section{Authorship statement}

All authors have contributed substantially to the conception of the work, the drafting of the manuscript and have approved the final version to be published and agree to be accountable for the work.

\section{References}

1. Engebretsen L, Risberg MA, Robertson B, et al. (2009) Outcome after knee dislocations: A 2-9 years follow-up of 85 consecutive patients. Knee Surg Sports Traumatol Arthrose 17 (9), 1013-1026.

2. Giannoulias CS, Freedman KB (2004) Knee dislocations: management of the multiligament-injured knee. Am J Orthop 33(11), 553-559.

3. Moatshe G, Chahla J, LaPrade RF, et al. (2017) Diagnosis and treatment of multiligament knee injury: State of the art. J ISAKOS 2(3), 152-161.

4. Stuart MJ (2001) Evaluation and treatment principles of knee dislocations. Oper Tech Sports Med 9(2), 91-95.
5. Berg EE (1995) Posterior cruciate ligament tibial inlay reconstruction. Arthroscopy 11(1), 69-76.

6. Jakob R, Rüegsegger M (1993) Therapy of posterior and posterolateral knee instability. Orthopade 22(6), 405-413.

7. Burks RT, Schaffer JJ (1990) A simplified approach to the tibial attachment of the posterior cruciate ligament. Clin Orthop Relat Res 254, 216-219.

8. Richter DL, Held M, Campos T, et al. (2020) The management of knee dislocations in the limited-resource setting. J Bone Joint Surg 10, 2106.

9. Lobenhoffer P, Gerich T, Bertram T, et al. (1997) Particular posteromedial and posterolateral approaches for the treatment of tibial head fractures. Unfallchirurg 100(12), 957-967.

10. Hoppenfeld S (2012) The Knee, in Surgical exposures in orthopaedics: The anatomic approach. Hoppenfeld S, DeBoer P, Buckley R, Editors. Lippincott Williams \& Wilkins, pp. 520526.

11. Gallagher J, Tierney P, Murray P, et al. (2005) The infrapatellar fat pad: Anatomy and clinical correlations. Knee Surg Sports Traumatol Arthrosc 13(4), 268-272.

12. Maculé F, Sastre S, Lasurt S, et al. (2005) Hoffa's fat pad resection in total knee arthroplasty. Acta Orthop Belg 71(6), 714.

13. Richter D, Wascher DC, Schenck RC (2004) A novel posteromedial approach for tibial inlay PCL reconstruction in KDIIIM injuries: Avoiding prone patient positioning. Clin Orthop Relat Res 472(9), 2680-2690.

14. Arciero RA (2005) Anatomic posterolateral corner knee reconstruction. Arthroscopy 21(9), 1141-1147.

15. LaPrade RF, Johansen S, Wentorf FA, et al. (2004) An analysis of an anatomical posterolateral knee reconstruction: An in vitro biomechanical study and development of a surgical technique. Am J Sports Med 32(6), 1405-1414.

16. Larsen MW, Moinfar AR, Moorman CT (2004) Posterolateral corner reconstruction-fibular-based technique. J Knee Surg 18 (02), 163-166.

17. Stannard JP, Brown SL, Robinson JT, et al. (2005) Reconstruction of the posterolateral corner of the knee. Arthroscopy 21(9), 1051-1059.

18. Frosch K-H, Balcarek P, Walde T, et al. (2010) A new posterolateral approach without fibula osteotomy for the treatment of tibial plateau fractures. J Orthop Trauma 24(8), 515520.

19. Bodendorfer BM, Keeling LE, Michaelson EM, et al. (2019) Predictors of knee arthrofibrosis and outcomes after arthroscopic lysis of adhesions following ligamentous reconstruction: A retrospective case-control study with over two years' average follow-up. J Knee Surg 32(06), 536-543.

20. Fanelli GC, Edson CJ (2012) Surgical treatment of combined PCL-ACL medial and lateral side injuries (global laxity): Surgical technique and 2- to 18-year results. J Knee Surg 25(4), 307-316.

21. Jazayeri SM, Esmaili Jah AA, Karami M (2009) A safe posteromedial approach to posterior cruciate ligament avulsion fracture. Knee Surg Sports Traumatol Arthrosc 17(3), 244-247.

22. Nicandri GT, Klineberg EO, Wahl CJ, et al. (2008) (2008) Treatment of posterior cruciate ligament tibial avulsion fractures through a modified open posterior approach: Operative technique and 12- to 48-month outcomes. J Orthop Trauma 22(5), 317-324. 
23. Trickey EL (1980) Injuries to the posterior cruciate ligament: Diagnosis and treatment of early injuries and reconstruction of late instability. Clin Orthop Relat Res 147, 76-81.

24. Boutefnouchet T, Bentayeb M, Qadri Q, et al. (2013) Long-term outcomes following single-bundle transtibial arthroscopic posterior cruciate ligament reconstruction. Int Orthop 37(2), 337-343.

25. Adler T, Friederich NF, Amsler F, et al. (2015) Clinical and radiological long-term outcome after posterior cruciate ligament reconstruction and nonanatomical popliteus bypass. Int Orthop 39(1), 131-136.

26. Hooper PO III, Silko C, Malcolm TL, et al. (2018) (2018) Management of posterior cruciate ligament tibial avulsion injuries: A systematic review. Am J Sports Med 46(3), 734 742 .

27. Levy DM, Erickson BJ, Bach BR (2018) Open versus arthroscopic anterior cruciate ligament reconstruction: A systematic review of randomized controlled trials. Curr Orthop Pract 28(5), 449-452.
28. Halinen J, Koivikko M, Lindahl J, et al. (2009) The efficacy of magnetic resonance imaging in acute multi-ligament injuries. Int Orthop 33(6), 1733-1738.

29. James EW, Williams BT, LaPrade RF (2014) Stress radiography for the diagnosis of knee ligament injuries: A systematic review. Clin Orthop Relat Res 472(9), 2644-2657.

30. LaPrade RF, Bernhardson AS, Griffith CJ, et al. (2010) Correlation of valgus stress radiographs with medial knee ligament injuries: An in vitro biomechanical study. Am J Sports Med 38(2), 330-338.

31. LaPrade RF, Heikes C, Bakker AJ, et al. (2008) The reproducibility and repeatability of varus stress radiographs in the assessment of isolated fibular collateral ligament and gradeIII posterolateral knee injuries: An in vitro biomechanical study. J Bone Joint Surg 90(10), 2069-2076.

32. Jackman T, LaPrade RF, Pontinen T, et al. (2008) Intraobserver and interobserver reliability of the kneeling technique of stress radiography for the evaluation of posterior knee laxity. Am $\mathrm{J}$ Sports Med 36(8), 1571-1576.

Cite this article as: Held M, Laubscher M, von Bormann R, Richter DL, Wascher DC \& Schenck RC (2021) Open approaches for cruciate ligament reconstruction in knee dislocations: A technical note and case series. SICOT-J 7, 17 